\title{
ASTROMETRIC PROGRAMS WITH THE NEW HAMBURG PLATE MEASURING MACHINE
}

\author{
CHR. DE VEGT, L. WINTER and N. ZACHARIAS \\ Hamburg Observatory \\ Gojenbergsweg 112 \\ 21029 Hamburg \\ Germany
}

\begin{abstract}
With the new Hamburg astrometric measuring machine, large sets of plates can be digitized very quickly with submicrometer accuracy. In particular about 2000 plates of the AGK2-catalog, mean epoch 1930, can be remeasured now for the first time to their limiting magnitude, about $B=11$. The new AGK2-data therefore will cover practically the whole AC-catalog and TYCHO-stars in the northern hemisphere.

All plates will be reduced using the HIIPPARCOS results as the reference frame when available in 1996. The new AGK2-data will provide a unique data base for the determination of high accuracy proper motions (about 2 mas/yr) of all TYCHO stars in the northem hemisphere. Furthermore, for the first time a dense reference frame for a final reduction of the Astrographic Catalog (AC) and the large deep sky surveys will be generated by this catalog. The inferior situation in the southern hemisphere will be addressed briefly.
\end{abstract}

\section{Introduction}

The new Hamburg plate measuring machine has been designed and optimized for high precision astrometric measuring programs. Based on a granite $x-y$ table, a large field CCD-camera is used to map the whole plate area in a frame-by-frame mode, where each frame covers about $60 \mathrm{~mm}^{2}$ at 1:1 magnification. Using also a dedicated VME-bus computer system with special vector processors, online data reduction can be achieved. Any plates of about $220 \times 220 \mathrm{~mm}^{2}$ typical measuring area can be measured to the plate limit and processed in about 1 hour (for further details see: de Vegt et al. 1992; Winter et al. 1992; Winter [this volume]). The remeasurement of important old plate material which typically contains some thousand plates therefore is now feasible.

\section{Measuring Programs}

Presently the machine is in its final test phase; routine operation is expected to begin in Fall ' 93 . Several measuring programs are in preparation and will be discussed subsequently. 


\subsection{A GROUNDBASED HIPPARCOS EXTRAGALACTIC LINK}

Now that the HIPPARCOS mission has finished the observational phase, data reduction is at full pace. A special subtask is the final transformation of the resulting rigid Hipparcos stellar net into the VLBI-based extragalactic reference frame. Besides other methods a groundbased link can be achieved by using deep plates of optical counterparts of these radio sources and determine their optical positions in the HIPPARCOS system via wide-field astrograph plates to provide faint reference stars for the deep object plates ( for details see: de Vegt 1992; de Vegt \& Johnston 1991).

Our present measuring program contains about 400 radio source fields over the whole sky which requires the measurement of some 1000 astrograph and deep object plates from many large telescopes.

At the same time, this program is part of a more general international research project to establish a global high precision radio-optical reference frame (de Vegt et al. 1991; Ma et al. 1990).

\subsection{THE AGK2-PLATE MATERIAL}

The successful measuring program of Hipparcos (about 120,000 brighter stars with positions, p.m. and parallaxes at the 2 mas accuracy level) also includes the TYCHO mission which will provide in addition about 1 million stars down to magnitude $B=11$ but with a reduced positional accuracy of about 30 mas and also precise B - V photometry but no p.m. On the northern hemisphere about 2000 plates of the AGK2-catalog contain nearly all TYCHO stars at average epoch 1930. All plates have 2 exposures $(3+10 \mathrm{~min}$.). However for the original AGK2-catalog only the $10 \mathrm{~min}$. exposure was measured and most of the faint stars were not contained in the program. The actual plate limit is close to $B=12$, thus providing a unique data material for the determination of precise p.m. for the TYCHO stars.

The remeasurement of these plates (both exposures) to the plate limit therefore has first priority in our measuring program.

The remeasurement requires a careful study of complex systematic errors depending on position in the plate area, magnitude and color (for a detailed discussion see: Dieckvoss \& Heckmann 1975; Eichhorn 1974). The original AGK2 results can be substantially improved now by using the Hipparcos results as the future reference frame which will supersede the original AGK2A reference frame by several orders. On each AGK2 $5 \times 5 \mathrm{deg}^{2}$ plate field about $50-70$ Hipparcos stars will be available for the plate reduction, which is at least twice the number of previously available AGK2A reference stars.

In addition, for the first time precise magnitude and colors for these stars will be available to set up a suitable plate reduction model which allows a statistically significant determination of all necessary color and magnitude dependent terms.

As the Hipparcos stars do not cover very well the faint end of the magnitudes, a complex block adjustment procedure, including part of the TYCHO stars, will be set up for the new reduction. The expected final positional accuracy of the new AGK2-catalog, based on both exposures, will be about 0.12 arcsec.

For all Tycho stars in the northern hemisphere, then, p.m. with an estimated accuracy of 2 mas/yr can be determined.

Concerning the required measuring time, about 1000 camera frames/plate with about $20 \%$ overlap will be necessary. Assuming a typical measuring and computing time of about 
$4 \mathrm{sec} / \mathrm{frame}$, a single plate (measuring area about $200 \times 200 \mathrm{~mm}$ ), measured in 2 orientations to compensate for a possible small magnitude equation of the measuring machine, can be processed therefore in less than 3 hours.

Depending on financial support for that project, the total remeasuring program can be accomplished in about $2-3$ years and will meet perfectly the time frame of the anticipated Tycho results (about 1997).

Unfortunately there is no similar solution in the southern hemisphere. A possible approach will be the combination of the CPC2 catalog with TYCHO, resulting in an estimated p.m. accuracy of about 3 mas/yr. However the limiting magnitude of the CPC2 is only close to B $=10$ (de Vegt et al. 1993).

\section{Remeasurement of Selected AC Zones}

Some zones have been measured originally only with moderate accuracy (Eichhorn 1974); an example is the Vatican zone (decl. +55 to +65 deg.). The accuracy of these zones can be improved considerable by remeasurement. Some plate material of the Vatican zone has been remeasured earlier on the visual MANN $422 \mathrm{~F}$ comparator at Hamburg Observatory in the fields of open star clusters and a factor 2 - 3 could be gained with respect to the original catalog data. The plates of these zones have 3 exposures $(6,3 \mathrm{~min}$., $30 \mathrm{sec}$.). Originally only the $3 \mathrm{~min}$. exposure was used as no long-screw measuring machine was available. Positions were interpolated by using a superimposed grid with known geometry (Eichhorn 1974).

A second important problem is the availability of a suitably dense reference frame for these plates, because the AC plate fields are $2 \times 2 \mathrm{deg}^{2}$ in general, and so the Hipparcos stars will not be sufficient. As has been discussed above, the TYCHO stars will serve as an optimal reference frame for the new reduction of the AC, provided a precise system of p.m. can be established globally to extrapolate the TYCHO positions to these early epochs (mainly 1900-1920).

From the remeasurement of the Vatican zone a final positional accuracy of 0.15 arcsec can be estimated. Globally, a re-reduction of all AC data may result in an average accuracy of about 0.2 arcsec (de Vegt 1991).

The Vatican plates are still in fairly good condition for remeasurement. In general the situation is very heterogeneous, plates of some zones are lost totally already. The Vatican zone contains about 1000 plates; a special measuring problem is posed by the superimposed gridlines which have to be treated by special algorithms. Measurement of a sample of test fields is scheduled for the end of this year. Due to the early epoch of this zone (mostly around 1900), p.m. with an accuracy of 1-2 mas/yr can be easily derived if these data are combined with suitable new epoch material.

\section{Conclusions}

The availability of the HIPPARCOS and TYCHO results in the anticipated 1996/1997 time frame will allow for the first time the provision of an uncompromised reduction of very important old epoch plate material, resulting in precise p.m. of several millions of stars for kinematic studies of our Galaxy in a magnitude range $(9-12)$ where other groundbased data are not very frequent. In addition this catalog material will also allow a suitable reduction of the very deep sky survey Schmidt plates to be performed where star images of presently available reference stars catalogs 
are mostly too overexposed to provide a satisfactory astrometric plate solution.

\section{References}

de Vegt, Chr., 1991. In 'Astrophys. Space Science', Proc. IAU Coll. 100, Belgrade 1987, 177, 3.

de Vegt, Chr., 1992. ESO Messenger, 69, 28.

de Vegt, Chr. and Johnston, K.J., 1991. Adv. Space Research, 11, 133.

de Vegt, Chr., Murray, A., Zacharias, N., Nicholson, W., Penston, M.J. and Clube, S.V.M., 1993. Astron. \& Astrophys. Suppl., 97, 985.

de Vegt, Chr., Winter, L. and Zacharias, N., 1992. In 'Digitised Optical Sky Surveys', eds. H.T. MacGillivray and E.B. Thomson, Kluwer, Dordrecht, p. 115.

Dieckvoss, W. and Heckmann, O., 1975. 'Introduction to AGK3', Hamburg.

Eichhorn, H., 1974. 'Astronomy of Star Positions', F. Ungar Publ.

Ma, C., Schaffer, D., de Vegt, Chr., Johnston, K.J. and Russell, J., 1990. Astron. J, 99, 1284.

Winter, L., this volume.

Winter, L., de Vegt, Chr. and Zacharias, N., 1992. In 'Digitised Optical Sky Surveys', eds. H.T. MacGillivray and E.B. Thomson, Kluwer, Dordrecht, p. 123. 